PREPARED FOR THE U.S. DEPARTMENT OF ENERGY, UNDER CONTRACT DE-AC02-76CH03073

PPPL-3887

PPPL-3887

UC-70

Turbulence Spreading into Linearly Stable Zone and Transport Scaling

by

T.S. Hahm, P.H. Diamond, Z. Lin, K. Itoh, and S.-I. Itoh

October 2003

NM|

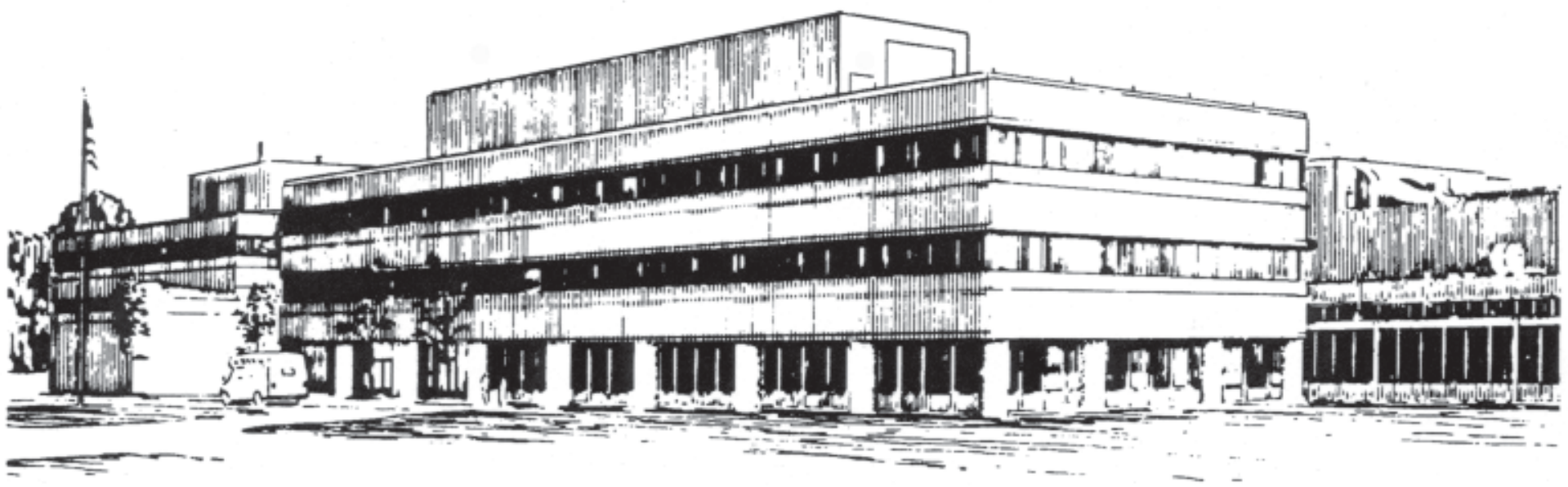

PRINCETON PLASMA PHYSICS LABORATORY PRINCETON UNIVERSITY, PRINCETON, NEW JERSEY 


\section{PPPL Reports Disclaimer}

This report was prepared as an account of work sponsored by an agency of the United States Government. Neither the United States Government nor any agency thereof, nor any of their employees, makes any warranty, express or implied, or assumes any legal liability or responsibility for the accuracy, completeness, or usefulness of any information, apparatus, product, or process disclosed, or represents that its use would not infringe privately owned rights. Reference herein to any specific commercial product, process, or service by trade name, trademark, manufacturer, or otherwise, does not necessarily constitute or imply its endorsement, recommendation, or favoring by the United States Government or any agency thereof. The views and opinions of authors expressed herein do not necessarily state or reflect those of the United States Government or any agency thereof.

\section{Availability}

This report is posted on the U.S. Department of Energy's Princeton Plasma Physics Laboratory Publications and Reports web site in Fiscal Year 2004. The home page for PPPL Reports and Publications is: http://www.pppl.gov/pub_report/

DOE and DOE Contractors can obtain copies of this report from:

U.S. Department of Energy

Office of Scientific and Technical Information

DOE Technical Information Services (DTIS)

P.O. Box 62

Oak Ridge, TN 37831

Telephone: (865) 576-8401

Fax: (865) 576-5728

Email: reports@adonis.osti.gov

This report is available to the general public from:

National Technical Information Service

U.S. Department of Commerce

5285 Port Royal Road

Springfield, VA 22161

Telephone: $1-800-553-6847$ or

(703) $605-6000$

Fax: (703) 321-8547

Internet: http://www.ntis.gov/ordering.htm 


\title{
Turbulence Spreading into Linearly Stable Zone and Transport Scaling
}

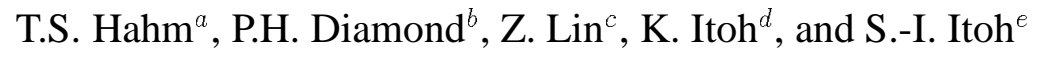 \\ ${ }^{a}$ Princeton University, Plasma Physics Laboratory, Princeton, NJ 08543, USA \\ ${ }^{b}$ University of California San Diego, La Jolla, CA 92093-0319, USA \\ ${ }^{c}$ University of California Irvine, Irvine, CA 92697, USA \\ ${ }^{d}$ National Institute for Fusion Science, Nagoya 464-01, Japan \\ ${ }^{e}$ Research Institute for Applied Mechanics, Kyushu University 87, Kasuga 816, Japan
}

October 9, 2003

\begin{abstract}
We study the simplest problem of turbulence spreading corresponding to the spatio-temporal propagation of a patch of turbulence from a region where it is locally excited to a region of weaker excitation, or even local damping. A single model equation for the local turbulence intensity $I(x, t)$ includes the effects of local linear growth and damping, spatially local nonlinear coupling to dissipation and spatial scattering of turbulence energy induced by nonlinear coupling. In the absence of dissipation, the front propagation into the linearly stable zone occurs with the property of rapid progression at small $t$, followed by slower $s u b$ diffusive progression at late times. The turbulence radial spreading into the linearly stable zone reduces the turbulent intensity in the linearly unstable zone, and introduces an additional dependence on the $\rho^{*} \equiv \rho_{i} / a$ to the turbulent intensity and the transport scaling. These are in broad, semi-quantitative agreements with a number of global gyrokinetic simulation results with zonal flows and without zonal flows. The front propagation stops when the radial flux of fluctuation energy from the linearly unstable region is balanced by local dissipation in the linearly stable region.
\end{abstract}

\section{Introduction}

Achieving understanding of turbulent transport is necessary for the design of an economical advanced 
tokamak fusion reactor. In recent years, progress in experiment, theory and computation has been dramatic, yet the 'Holy Grail' of predictive capacity by other than brute-force, case-by-case direct numerical simulation, remains elusive. Serious challenges remain due to the fact that virtually all models of fluctuation levels and turbulent transport are built on an assumption of local balance of linear growth with linear damping and nonlinear coupling to dissipation. Here, 'local balance' refers to balance at a point or in a region comparable in extent to the modal width. Such models thus necessarily exclude mesoscale dynamics which refers to dynamics on scales larger than a mode or integral scale eddy size, but smaller than the system size or profile scale length. In particular, transport barriers, avalanches, heat and particle pulses all are mesoscale phenomena[1,2,3].

In this paper, we identify and study in depth the simplest, most minimal problem in the mesoscale dynamics category before proceeding to consider more complicated examples. In this case, the 'minimal problem' is that of the spatio-temporal propagation of a patch of turbulence from a region where it is locally excited to a region of weaker excitation, or even local damping. This process can be described by a single model equation for the local turbulence intensity $I(x, t)$, which includes the effects of local linear growth and damping, spatially local nonlinear coupling to dissipation and spatial scattering of turbulence energy induced by nonlinear coupling. These effects combine to give an energy equation loosely of the form

$$
\frac{\partial I}{\partial t}-\frac{\partial}{\partial x} \chi(I) \frac{\partial I}{\partial x}=\gamma(x) I-\alpha I^{1+\beta},
$$

the terms of which correspond to nonlinear spatial scattering (i.e. typically $\chi(I) \sim \chi_{0} I^{\beta}$ where $\beta=1$ for weak turbulence, and $\beta=1 / 2$ for strong turbulence), linear growth and damping, and local nonlinear decay, respectively. Here $\alpha$ is a nonlinear coupling coefficient. Note that $\alpha$ and $\chi_{0}$ could be functions of radius. This energy equation is the irreducible minimum of the model, to which additional equations for other fields, and contributions to dynamics which feedback on $I$, may be added. Note that the above energy equation manifests the crucial effect of spatial coupling in the nonlinear diffusion term. This implies that the integrated fluctuation intensity in a region of extent $\triangle$ about a point $x$ (i.e. $\left.\int_{x-\triangle}^{x+\triangle} I\left(x^{\prime}\right) d x^{\prime}\right)$ can grow, even for negative $\gamma(x)$, so long as $\chi(I) \partial I /\left.\partial x\right|_{x-\triangle} ^{x+\triangle}$ is sufficiently large. Alternatively, $I$ can decrease, even for positive $\gamma(x)$, should $\chi(I) \partial I /\left.\partial x\right|_{x-\triangle} ^{x+\triangle}$ be sufficiently negative. Thus, the profile of fluctuation intensity is crucial to its spatio-temporal evolution. These simple observations nicely illustrate the failure of the conventional local saturation paradigm[4], and strongly support the argument that propagation of turbulence energy is a crucial, fundamental problem in understanding confinement scalings for fusion devices in which growth and damping rate profiles vary rapidly in space.

While the radial spreading of turbulence has been widely observed in previous global nonlinear gyroki- 
netic and mode coupling simulations[5, 6, 7], its significance has not been widely recognized. Its effect on turbulent transport scaling has been addressed only recently[8]. In that work[8], we conjectured that the turbulence radial spreading into the linearly stable zone can reduce the turbulent intensity in the linearly unstable zone, and introduce an additional dependence on the $\rho^{*} \equiv \rho_{i} / a$ to the turbulent intensity which is otherwise determined by local physics. Since the ion thermal diffusivity $\chi_{i}$ was observed to be proportional to $I$ for the weak turbulence case in the previous gyrokinetic simulation[9], this, in turn, can cause a deviation from gyroBohm transport scaling which was expected from the local turbulence characteristics (i.e., a radial correlation length about $\Delta r \simeq 7 \rho_{i}$, independent of the system size). The basic features of analytic, dynamical model of turbulence[10] has been previously published in Ref. [11], In this paper, we present a more complete version and more detailed comparions to gyrokinetic simulation results[8].

The remainder of this paper is organized as follows. In Section 2, we propose a nonlinear diffusion equation as the simplest model for the problem of turbulence spreading, and present detailed analytic solutions of this nonlinear diffusion equation. Sec.3 contains the effects of radial spreading on transport scaling and comparisons to recent global gyrokinetic simulation results. Sec.4 consists of conclusions.

\section{Dynamics of Turbulence Spreading}

Another aspect of the dynamics which falls outside the traditional "local balance" paradigm of Kadomtsev, et al.[4] is illustrated by the equation for $I(x)$. First, turbulence energy propagation is intrinsically non-diffusive, since $\chi(I)$ increases with $I$. This is easily seen by observing that for turbulent diffusion, $\chi(I)=\chi_{0} I^{\beta}$, so that the natural diffusive scalings for the width of a turbulent patch are $\ell^{2} \sim \chi_{0} I^{\beta} t$

and $I \ell=Q_{0} \equiv I_{0} \ell_{0}$. It thus follows that the self-similarity variable is $x / \ell(t)=x /\left(\chi_{0} Q_{0}^{\beta} t\right)^{1 /(2+\beta)}$, so a turbulent patch spreads as $\triangle x \sim\left(\chi_{0} Q_{0}{ }^{\beta} t\right)^{1 /(2+\beta)}$ in the absence of growth or dissipation. Contrary to conventional wisdom, this actually corresponds to sub-diffusive propagation, which has the property of rapid progression at small $t$, followed by slower progression at late times. Thus, the rapid re-adjustment and spatial spreading of turbulence intensity profiles observed in several gyrokinetic particle simulations $[5,6,8]$ are quite likely symptoms of turbulence propagation.

Focusing on the role of nonlinear diffusion in the weak turbulence regime (with $\beta=1$ in Eq. (1)) as observed in the gyrokinetic simulations $[8,9]$, we begin our analysis using the following nonlinear partial differential equation:

$$
\frac{\partial}{\partial t} I=\gamma(x) I-\alpha I^{2}+\chi_{0} \frac{\partial}{\partial x}\left(I \frac{\partial}{\partial x} I\right)
$$

Here $I \equiv\left\langle\left(\frac{e \phi}{T_{e}}\right)^{2}\right\rangle$ is the envelope of the turbulence intensity in the energy containing range in $k_{y}$ exclud- 
ing the zonal flows with $k_{y}=k_{z}=0, \gamma(x)$ is the "local" excitation rate of the instability, for instance the ion temperature gradient (ITG) driven mode, $x$ is a radial coordinate, $\alpha$ is the local nonlinear coupling term[12]. For a diffusion coefficient of turbulence $\chi_{i}$, we take $\chi_{i} \equiv \chi_{0} I$ with an explicit proportionality to $I$ as observed in the previous global nonlinear gyrokinetic simulation[9]. We note that Eq. (2) is similar to a generalized $K-\epsilon$ model for its direct inverse process, namely transport barrier propagation and broadening $[13,14,15]$.

First we review the local solution. In the absence of the nonlinear radial diffusion given by the last term, we can integrate Eq. (2) in time with an initial profile $I(x, 0)<\frac{\gamma(x)}{\alpha}$ to obtain,

$$
I(x, t)=\frac{\gamma(x)}{\alpha} /\left(1+\frac{\frac{\gamma(x)}{\alpha}-I(x, 0)}{I(x, 0)} e^{-\gamma(x) t}\right) .
$$

Eq. (3) describes the time evolution of the intensity towards a nonlinear saturation. In the region $\gamma>0$, $I$ initially grows exponentially with a linear growth rate $\gamma(x)$, then saturates at a finite level given by $\frac{\gamma(x)}{\alpha}$. However in the region where $\gamma<0$, this local solution predicts that the fluctuation vanishes $(I \rightarrow 0)$.

Now we study in detail how the nonlinear diffusion term in Eq. (2) allows fluctuations to spread into a zone where $\gamma<0$. In the region where $\gamma(x) \simeq 0$ and $I<<1$, Eq. (2) simplifies to the following nonlinear partial differential equation which is also known as the modified porous-medium equation[16].

$$
\frac{\partial}{\partial t} I_{0}=\chi_{0} \frac{\partial}{\partial x}\left(I_{0} \frac{\partial}{\partial x} I_{0}\right)
$$

We consider a smooth radially varying linear excitation rate profile $\gamma(x)$ which is similar in shape to the ones used in a global gyrokinetic simulation of ITG instablities discussed in Refs. [5, 8] in shape. As shown in Fig. $1, \gamma>0$ in the middle for $\left|x-x_{i}\right|<W, x_{i}$ is the position where $\gamma(x)$ is maximum.

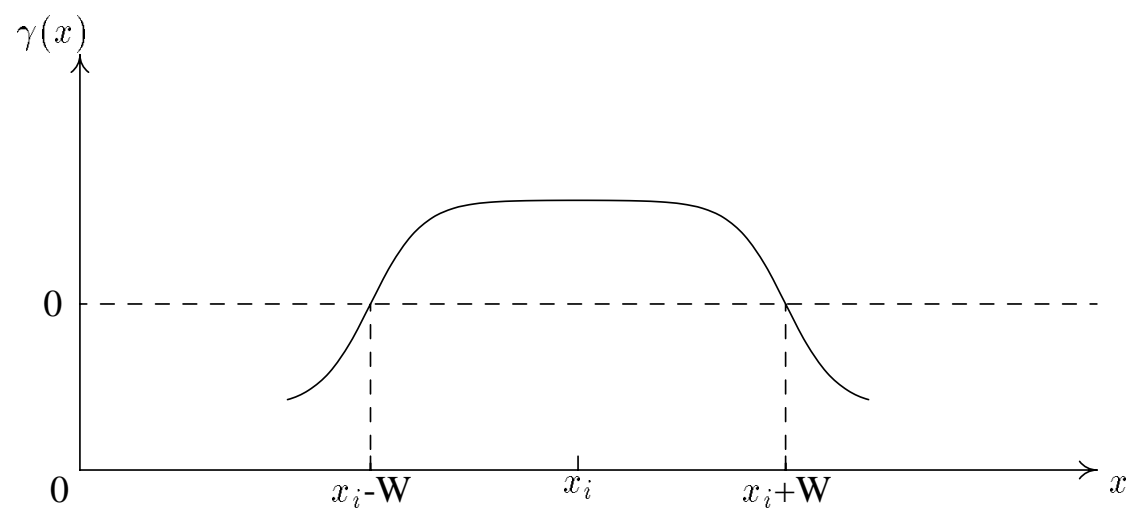

Figure 1: Local excitation rate $\gamma(x)$ as a function of radius.

Then $\gamma$ decreases monotonically towards the axis and the edge, becomes 0 at $x=x_{i}-W$ and at $x=$ $x_{i}+W$, and becomes negative for $\left|x-x_{i}\right|>W$. In this paper, we only consider the case where the 
background pressure and $\gamma(x)$ do not change in time. This simple case is still relevant for turbulence dynamics which occurs in a time scale shorter than transport time scale. This also makes comparisons to the recent gyrokinetic simulations[11] more straightforward. For more challenging problems such as the formation of transport barriers where the disparity in time scales becomes less obvious, one needs to extend the theory to a multifield nonlinear system $[14,15,17,18]$ in which evolutions of $\mathbf{E} \times \mathbf{B}$ flows and the pressure gradient are included. Gyrokinetic simulation in Ref.[11] shows that the volume integrated fluctuation intensity saturates in magnitude as the self-generated zonal flows grows to a saturated amplitude as discussed in detail in Refs.[19,20]. Then, the radial spreading of turbulence occurs from approximately $t=8 / \gamma$, while the volume integrated fluctuation intensity stays approximately a constant in time as shown in Fig. 2. We focus our studies on this later phase.

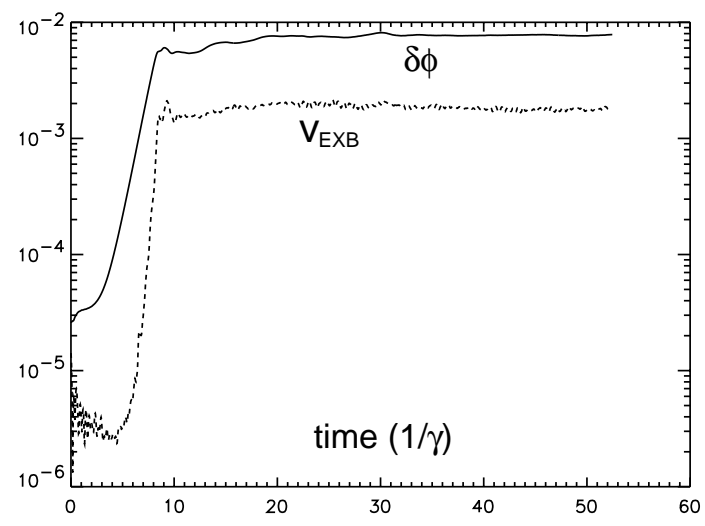

Figure 2: The volume integrated fluctuation intensity $(\delta \phi)$ saturates in magnitude as the self-generated zonal flows $\left(V_{\mathbf{E} \times \mathbf{B}}\right)$ grows to a saturated amplitude. The radial spreading of turbulence occurs from approximately $t=8 / \gamma$, while the volume integrated fluctuation intensity stays approximately a constant in time.

For an initial profile $I_{0}(x, 0)=\frac{\epsilon}{W}\left(1-\frac{\left(x-x_{i}\right)^{2}}{W^{2}}\right) H\left(W-\left|x-x_{i}\right|\right)$, Eq. (4) has an exact solution[16]:

$$
I_{0}(x, t)=\frac{\epsilon}{\left(6 \epsilon \chi_{0} t+W^{3}\right)^{1 / 3}}\left(1-\frac{\left(x-x_{i}\right)^{2}}{\left(6 \epsilon \chi_{0} t+W^{3}\right)^{2 / 3}}\right) H\left(W-\left|x-x_{i}\right|\right),
$$

where $\epsilon$ is the volume-integrated intensity, $H$ is a Heavyside function. Eq. (5) shows that in the absence of linear or nonlinear damping (the first term and the second term on the RHS of Eq. (2)), a fluctuation front at $x=x_{i}+\left(W^{3}+6 \epsilon \chi_{0} t\right)^{1 / 3}$ will propagate beyond $x_{0} \equiv x_{i}+W$ indefinitely. (The same comment applies to another front at $x=x_{i}-W$ which propagates to the left.) The time evolution of $I_{0}$ exhibiting a localized front-like solution described by Eq. (5) is plotted in Fig. 2. 


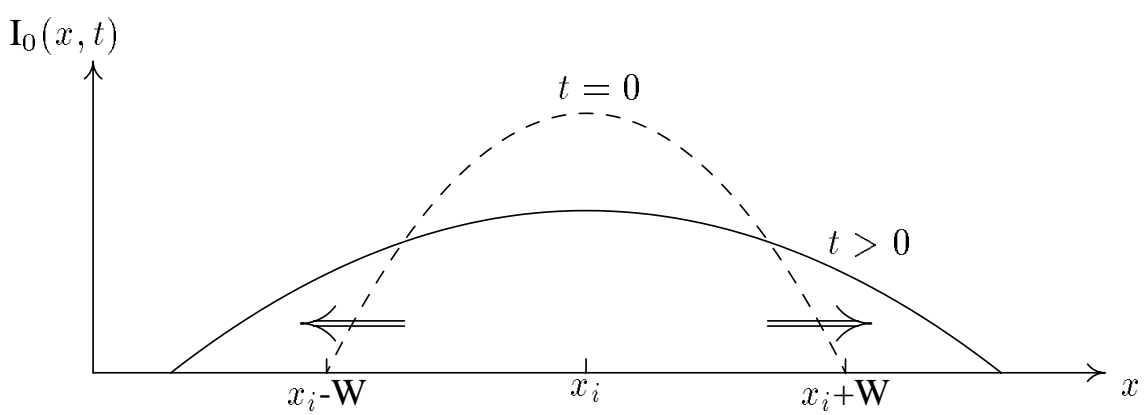

Figure 3: Eq.(5) describes a propagating localized front-like solution.

We note that the short time behavior of propagation can be characterized by $\left(x-x_{0}\right)_{\text {front }} \simeq U_{x} t$ after expanding in $\left(x-x_{0}\right)_{\text {front }} / W$, with $U_{x}=\frac{2 \epsilon \chi_{0}}{W^{2}}$. It is obvious that the expression for $U_{x}$ from our nonlinear theory is qualitatively different from the radial group velocity of a drift wave. This is one of signatures which distinguish our nonlinear diffusion theory from the other models which heavily rely on the specific properties of the drift wave linear dispersion relation[7], or on nonlinear enhancement of dispersion[21] of the 4-mode system consisting of toroidal eigenmodes and zonal flows[22]. The long term behavior of propagation is sub-diffusive, as discussed previously,

$$
\triangle x \sim\left(\chi_{0} Q_{0} t\right)^{1 / 3}
$$

The relevant question here is what is the physics mechanism responsible for a saturation of the fluctuation spreading in a linearly stable zone? At a conceptual level, most existing models of turbulent transport are based on a local balance of excitation and dissipation. For instance, the commonly used local saturation condition á la Kadomtsev[4] in which one balances the linear growth rate $\gamma>0$ and the local nonlinear damping (i.e., $\gamma \sim k_{\perp}^{2} D_{\text {turb }}$ ) does not apply here. We expect that the fluctuation front would cease to propagate if the fluctuation energy flux due to radial propagation spread into the linearly stable zone and was balanced by dissipation. First, we consider the case where the linear damping near the propagating front $\left(\gamma(x) \simeq-\left|\gamma^{\prime}\right|\left(x-x_{0}\right)\right)$ is strong enough to play a dominant role in limiting the radial spreading. The scaling for $\Delta$ can be obtained by balancing the time required for linearly damping the fluctuation at $x=x_{0}+\Delta$, i.e., $T_{\text {damp }} \simeq \frac{1}{\left|\gamma^{\prime}\right| \Delta}$, against the time required for the front to propagate a distance $\Delta$ (which is shorter than the system size), i.e., $T_{\text {prop }} \simeq \frac{\Delta}{U_{x}}$. The resulting scaling with respect to the damping rate is weaker $\left(\Delta \propto\left|\gamma^{\prime}\right|^{-1 / 2}\right)$ than that based on a heuristic argument based on the linear toroidal couping, $\Delta \propto \gamma_{\text {damp }}^{-1}$ [23]. Quantitatively, the front stops propagating when the width of spreading $\Delta$ satisfies the following condition:

$$
\frac{\partial}{\partial T} \int_{x_{0}}^{x_{0}+\Delta} d x I_{0}(x, T)=-\int_{x_{0}}^{x_{0}+\Delta} d x \gamma(x, T) I_{0}(x, T),
$$


which yields the expression for the width of the radial spreading, $\Delta$ :

$$
\Delta^{2} \simeq \frac{12 \epsilon \chi_{0}}{\left|\gamma^{\prime}\right| W^{2}}
$$

As expected, higher fluctuation intensity in a linearly unstable zone $(\epsilon)$ enhances the radial spreading, while a strong linear damping reduces it.

We note that depending on the scaling of ion thermal transport in the absence of turbulence spreading, $\chi \propto \epsilon \chi_{0} / W$, Eq. (8) predicts different scalings of the radial spreading $\Delta$, with respect to the system size. These are in broad semi-quantitative agreements with observations from several global gyrokinetic simulation results $[5,6,8,24,25]$. We discuss details in the next section. If the linear damping is sufficiently weak, the nonlinear coupling to damped modes in the dissipative range could act to limit the range of turbulence spreading in space. The nonlinear damping is a manifestation of the nonlinear mode coupling in $\mathrm{k}$-space including the interaction with zonal flows[22, 26, 27]. However, the incoherent noise, which is ignored in our nonlinear diffusion model in this paper, can also be produced from nonlinear mode coupling and will likely to significantly enhance the range of spreading, in particular near low order rational surfaces. This effect will be studied in the future publication. With this caveat in mind, our estimation below which relies on the coherent nonlinear mode coupling only, should be taken as an underestimate of turbulence spreading. Following a straightforward dimensional analysis, we find that $\Delta \propto \sqrt{\frac{\chi_{0}}{\alpha}}$, when the nonlinear damping saturates the fluctuation propagation.

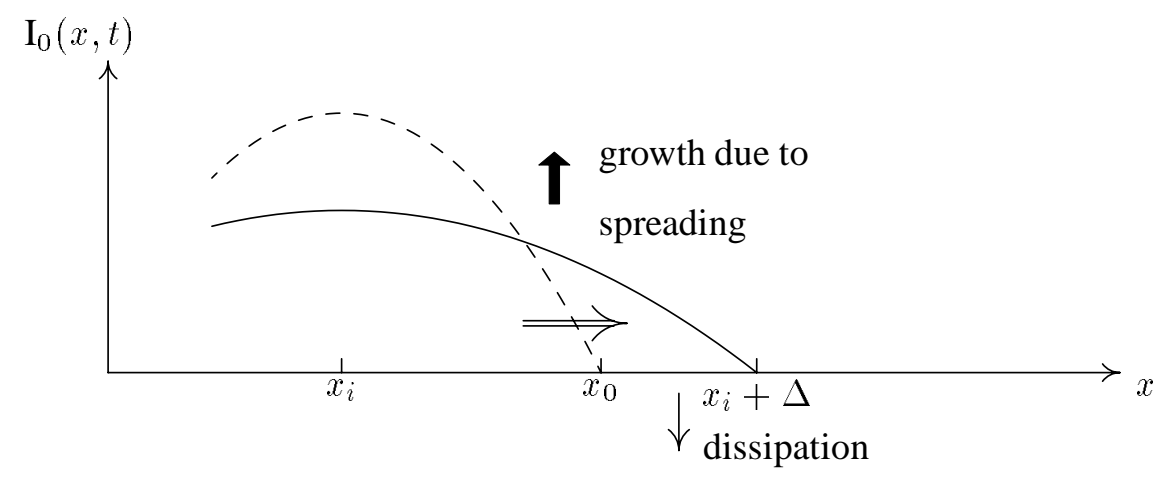

Figure 4: Fluctuation front ceases to propagate if the fluctuation energy

flux due to radial propagation is balanced by dissipation.

\section{Effects of Turbulence Spreading on Transport Scalings}

While the radial spreading of turbulence has been widely observed in the previous global nonlinear simulations[7, $6,5,24,25]$, its effect on turbulent transport scaling has not been addressed until recently[8]. In that work[8], 
we conjectured that the radial turbulence spreading into the linearly stable zone can reduce the turbulent intensity in the linearly unstable zone, and introduce an additional dependence on the $\rho^{*} \equiv \rho_{i} / a$ to the turbulent intensity which is otherwise determined through a local physics. Since $\chi_{i} \propto I$ for the weak turbulence case as observed in the previous gyrokinetic simulations[9], this, in turn, can cause a deviation from the gyroBohm transport scaling which was expected from the local turbulence characteristics (i.e., $\Delta r \simeq 7 \rho_{i}$ ) only. The basic features of analytic dynamical model of turbulence spreading[10] and progress toward a theoretical underpinning has been published in Ref. [11,28]. In this section, we present the specific predictions from our present nonlinear diffusion model and compare them to the recent gyrokinetic simulation results[29]. Eq. (5) shows that in the absence of dissipation, a fluctuation front movement into the linearly stable zone by a radial width $\Delta$ reduces the peak fluctuation intensity at $x=x_{i}$ to

$$
I\left(x_{i}, T\right)=I\left(x_{i}, 0\right) /\left(1+\frac{\Delta}{W}\right) .
$$

Since we are interested in the regime where $\chi_{i} \propto I$ [9], we obtain

$$
\chi_{i}=\chi_{i 0} /\left(1+\frac{\Delta}{W}\right)
$$

where $\chi_{i 0}$ is the ion thermal diffusivity in the absence of the radial spreading of turbulence.

Based on the following estimation, we now argue that the reduction of the peak fluctuation intensity due to dissipation without the radial spreading of fluctuations is exponentially small in the limit $W / \rho_{i}>>1$. We seek a steady state solution of Eq. (2) by for a $\gamma(x)$ profile which is piece-wise constant in radius,

$$
-\chi_{0} \frac{\partial}{\partial x}\left(I \frac{\partial}{\partial x} I\right)=\gamma(x) I-\alpha I^{2}
$$

where $\gamma(x)=\gamma_{0}>0$, for $\left|x-x_{i}\right|<W$ and $\gamma(x)=-\left|\gamma_{d}\right|<0$, for $\left|x-x_{i}\right|>W$. One finds that for a piece-wise constant $\gamma$ profile, Eq. (11) can be written as a perfect derivative in $x$ by multiplying both sides by $I \frac{\partial I}{\partial x}$.

$$
-\frac{\chi_{0}}{4} \frac{\partial}{\partial x}\left(\frac{\partial}{\partial x} F\right)^{2}=\frac{2}{3} \gamma \frac{\partial}{\partial x} F^{3 / 2}-\frac{\alpha}{2} F^{2},
$$

here $F=I^{2}$. An integration of Eq. (12) in radius with boundary conditions $\frac{\partial}{\partial x} I(x)=0$, at $x=x_{i}$ and $I(x)=0$ for $\left|x-x_{i}\right| \rightarrow \infty$ guarantees a steady state solution for a large enough system size. Here, we estimate the reduction of peak turbulence intensity (at $x=x_{i}$ ) due to a dissipation at $\left|x-x_{i}\right|>W$ in the absence of radial spreading. By taking a limit of very strong linear damping $\left|\gamma_{d}\right| \rightarrow \infty$, we obtain the following relation by integrating Eq. (12) from $x=x_{i}$ to $x$,

$$
\frac{\chi_{0}}{4}\left(\frac{\partial}{\partial x} F\right)^{2}=\frac{2}{3} \gamma_{0}\left(F\left(x_{i}\right)^{3 / 2}-F^{3 / 2}\right)-\frac{\alpha}{2}\left(F\left(x_{i}\right)^{2}-F^{2}\right) .
$$


By integrating Eq. (13) in $F$ from $F\left(x_{i}+W\right)=0$ to $F\left(x_{i}\right)$ after undoing the square, we obtain the following integral relation between $W / \Delta r$ and $F\left(x_{i}\right)$,

$$
\int_{0}^{F\left(x_{i}\right)} \frac{d F}{\sqrt{\frac{8 \gamma_{0}}{3 \chi_{0}}\left(F\left(x_{i}\right)^{3 / 2}-F^{3 / 2}\right)-\frac{2 \alpha}{\chi_{0}}\left(F\left(x_{i}\right)^{2}-F^{2}\right)}}=W
$$

here we used the fact that $I(x)=0$ at $\left|x-x_{i}\right|=W$. A simple inspection shows that the integral exhibits a logarithmic divergence for $W \rightarrow \infty$ limit in which the fluctuation intensity saturation level is determined by the local physics, that is, $F\left(x_{i}\right) \simeq \gamma_{0} / \alpha$. An asymptotic analysis yields that

$$
F\left(x_{i}\right) \simeq \frac{\gamma_{0}}{\alpha}(1-O(\exp (-W / \Delta r)))
$$

where $\Delta r \equiv \sqrt{\chi_{0} / \alpha}$ is the radial correlation length which scales with the ion gyroradius. We, therefore, conclude that the reduction of intensity in the linearly unstable zone at the middle should originate mainly from the fluctuation spreading.

Having established a relation between the radial spreading and transport scaling in Eq. (10), we discuss the observations from previous global gyrokinetic simulations and possible relevance of our theoretical predictions to these simulations[5, 6, 8]. To our knowledge, the first significant numerical study addressing turbulence spreading has been performed in the context of a global mode couping analysis of toroidal drift wave[7]. It was observed that the linear toroidal coupling of different poloidal harmonics played a dominant role in the convective propagation of fluctuations into a region with a zero level background of fluctuations in most parameter regimes. It is worthwhile to note that Ref. [7] was published before the important role of the self-generated zonal flows in regulating turbulence in toroidal geometry was fully realized[19]. In a similar fashion to the mean $\mathbf{E} \times \mathbf{B}$ flow shear causes decorrelation of turbulence in the radial direction[30, 31], the random shearing by zonal flows[20, 26] which has not been included in Ref.[7], would make the linear toroidal coupling much weaker. This is shown by the measured reduction in the radial correlation length of fluctuations[20] as radially global toroidal eigenmodes get destroyed by the zonal flows in gyrokinetic simulations[19]. Ref. [7] reports that, when the linear toroidal coupling is suppressed, the fluctuation propagation due to nonlinear mode coupling has been observed to be close to a diffusive process. If we consider the limited numerical accuracy of the time of flight measurements employed in Ref. [7], this result does not seem to contradict our prediction of subdiffusive propagation as discussed at the beginning of Sec. II [23]. In the strong turbulence regime considered in Ref. [7] where $\beta=1 / 2$, we get $\triangle x \sim\left(\chi_{0} Q_{0}{ }^{1 / 2} t\right)^{2 / 5}$ which is difficult to differentiate from the diffusive behavior of $\triangle x \propto t^{1 / 2}$.

We expect that in the presence of zonal flows, the nonlinear radial diffusion remains a robust mechanism responsible for the turbulence spreading while the communication between different poloidal harmonics become relatively ineffective. Now we briefly describe a recent nonlinear gyrokinetic particle-in-cell 
simulations $[8,11]$ where the self-generated zonal flows regulate turbulence. These nonlinear gyrokinetic particle-in-cell simulations have been performed with Gyrokinetic Turbulence Code (or GTC) code[19]. Global field line following coordinates have been used to take advantage of fluctuation structures which align with equilibrium magnetic field lines. However, unlike many quasi-local codes in flux-tube geometry, GTC does not rely on the ballooning mode formalism which becomes dubious in describing meso-scale structures[1, 2, 3] in fully developed turbulence. Therefore, realistic radial profile variations can be included straightforwardly.

The simulation parameters used are based on those from Ref.[32] which uses a simple characterization of a typical DIII-D H-mode core plasma. A system size (or $\rho_{*}$ ) scan is then carried out with other dimensionless parameters fixed with a radial variation of ion temperature gradient included as shown in Ref. [11]. The peak value of $R / L_{T i}$ in the middle is 6.9 which is well above marginality. Towards the axis and the edge the gradient gets weaker. While the gyrokinetic particle simulations in the mid-90's[6, 5, 24, 25] showed that radially elongated eddys, which are closely related to the global toroidal eigenmodes, partially survived in the nonlinear phase, more recent global simulations showed that self-generated zonal flows break up the radially elongated eddys[19] and isotropize the $\mathrm{k}$ spectrum of fluctuations. The broadening in the $k_{r}$ spectrum of turbulence[20] in the presence of zonal flows in the simulation is a consequence of random shearing $[20,26,33]$. Therefore we think it is reasonable to expect that linear toroidal coupling effects become weaker in the presence of zonal flows. More detailed two point correlation analysis of simulation data indeed shows that the correlation length of fluctuations scales with ion gyroradius $\Delta r \simeq 7 \rho_{i}$, and these are invariant with respect to the system size. In the nonlinear phase of simulations, fluctuations spread radially. Their radial extent is approximately $25 \rho_{i}$ or $3 \sim 4$ radial correlation lengths in each direction. Interestingly, it is independent of the system size, as inferred from Fig. 5[29].

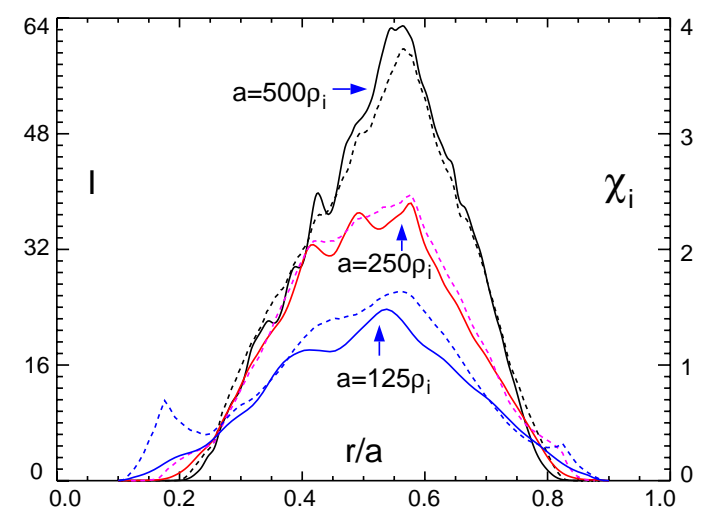

Figure 5: Fluctuation intensity profiles ( $I$ in solid lines) and ion thermal diffusivity ( $\chi_{i}$ in dotted lines), both in gyro-Bohm unit after nonlinear saturation for $a / \rho_{i}=125,250$, and 500: As the system size gets 
larger, the extent of radial spreading of turbulence into the linearly stable zone gets narrower relative to the system size. It scales with the ion gyroradius $\sim 25 \rho_{i}[29]$.

Using the values of $W, \epsilon, \chi_{0}, \gamma^{\prime}$ and $\alpha$ from simulations, we estimate the predicted scalings and values of $\Delta$ given in Eq. (8). From the simulations described above, $W=x_{0}-x_{i}=0.75 a-0.5 a=0.25 a$, $\chi_{i}(0.5 a, T)=1.35 \frac{c T_{i}}{\epsilon B} \frac{\rho_{i}}{L_{T i}}$, and $\gamma\left(x_{i}\right)=0.033 \frac{v_{T i}}{L_{T i}}$ for $k_{\theta} \rho_{i}=0.2$ where $I$ peaks at nonlinear saturation. Note that 0.033 is rather a small coefficient coming from kinetic effects not captured in a heuristic dimensional argument based on a simple fluid picture. We note that radial diffusion of ion heat is not always same as that of fluctuation intensity. For the estimation of $\gamma^{\prime}$, we use a scaling that $\gamma \propto k_{\theta} \rho_{i} \frac{v_{T i}}{R_{0}}\left(\frac{R_{0}}{L_{T i}}-\frac{R_{0}}{L_{c r i t}}\right)$ for small $k_{\theta} \rho_{i}$ near the local threshold as suggested by the results from the local ballooning calculation using the FULL code[34]. For these values mentioned above, Eq. (8) yields $\Delta \simeq 18 \rho_{i}$, in the rough range of fluctuation spreading observed in our simulation, $\Delta \simeq 25 \rho_{i}$. Considering the simplicity of our nonlinear model, this level of agreement is encouraging. If we use the value of $\Delta$ from the simulation, Eq. (10) based on our simple one dimensional theory yields,

$$
\chi_{i} \propto \chi_{g y r o B o h m} /\left(1+100 \rho_{*}\right)
$$

which is in rough agreement with the scaling trend observed in the simulations [8]. We also note that a similar argument based on Eq. (10) can also be used as a possible explanation for the previous observation of the "worse than Bohm transport scaling" from an early global gyrokinetic simulation[6, 25]. In this simulation without zonal flows, the radially global toroidal modes with radail width $\Delta r \propto \sqrt{W \rho_{i}}$ can easily lead to Bohm scaling of transport in an ideal plasma without the radial spreading of turbulence. Our theoretical results in Eq. (8) indicate that the width of spreading $\Delta \propto \sqrt{W \rho_{i}}$ due to $\Delta r \propto \sqrt{W \rho_{i}}$, although there has not been a systematic scaling study of the radial spreading in the absence of zonal flows. Following the same logic, one could deduce that $\chi_{i} \propto \chi_{B o h m} /(1+\Delta / W)$ due to the radial spreading of turbulence, and the resulting transport scaling is worse than Bohm. The observation of the fluctuation radial spreading in this simulation also suggests that while the zonal flows play a crucial role in regulating turbulence and in making linear toroidal couping weaker, they don't seem to play a direct role in either causing or inhibiting the radial spreading. Another independent global gyrokinetic simulation[5] in the presence of weak zonal flow shear, has reported a trend that transport scaling is close to Bohm, but tends to change very slowly from Bohm to gyroBohm as the system size gets larger. This intermediate result from a simulation which stands between the simulation of Ref.[8] with strong zonal flow shear and the simulation of Ref.[6] without zonal flows, is also in qualitative agreement with a hypothesis that the radial spreading of turbulence can change the transport scaling by increasing the volume of the active turbulence zone, and decreasing the local 
fluctuation intensity in the region where the instability is linearly strong.

\section{Conclusions}

In this paper, we have identified and studied in depth, the simplest, most minimal problem of turbulence spreading corresponding to the spatio-temporal propagation of a patch of turbulence from a region where it is locally excited to a region of weaker excitation, or even local damping. This process is described by a single model equation for the local turbulence intensity $I(x, t)$, which includes the effects of local linear growth and damping, spatially local nonlinear coupling to dissipation and spatial scattering of turbulence energy induced by nonlinear coupling. The principal results of this paper are as follows:

i) In the absence of dissipation, the front propagation into the linearly stable zone occurs with the property of rapid progression at small $t$, with a characteristic speed $U_{x}=\frac{2 \epsilon \chi_{0}}{W^{2}}$, followed by slower sub-diffusive progression at late times with $\triangle x \sim\left(\chi_{0} Q_{0} t\right)^{1 / 3}$.

ii) The turbulence radial spreading into the linearly stable zone reduces the turbulent intensity in the linearly unstable zone, and introduces an additional dependence on the $\rho^{*} \equiv \rho_{i} / a$ to the turbulent intensity which is otherwise determined by local physics. Since $\chi_{i}$ scales with the turbulent intensity $I$, this, in turn, can cause a change in the transport scaling with respect to $\rho^{*}$. These are in broad, semi-quantitative agreements with a number of global gyrokinetic simulation results with strong zonal flows[8], with weak zonal flows[5], and without zonal flows[6].

iii) The front propagation stops when the radial flux of fluctuation energy from the linearly unstable region is balanced by local dissipation in the linearly stable region. This work provides a new rule of thumb for determining a local fluctuation amplitude in the linearly stable zone.

It is worthwhile to note that the radial spreading of turbulence discussed here could play a role in tokamak plasmas. For instance, large $\chi_{e}$ as observed with the weak gradients in the core of RS (reversed shear) plasmas[35, 36, 37], is difficult to reconcile with the conventional linear stability based picture in which a non-zero fluctuation level is expected only in the region where the linear growth rate is positive.

\section{Acknowledgments}

The authors would like to thank X. Garbet, P. Ghendrih, and Y. Sarazin for useful conversations regarding their numerical simulation results. A part of work was performed at the Festival de Théorie, 2003 in Aixen-Provence, France. This work was supported by the U.S. Department of Energy Contract No. DE-AC02- 
76-CHO-3073 (PPPL), Grant number FG03-88ER 53275 (UCSD), Grant number DE-FG03-94ER54271 (UCI), the US DOE SciDAC plasma microturbulence project, the Grant-in-Aid for Scientific Research of MEXT, Japan, and by the collaboration programs of NIFS and of RIAM, Kyushu Univ.

\section{References}

[1] P.H. Diamond and T.S. Hahm, Phys. Plasmas, 2, 3640 (1995).

[2] S.-I. Itoh and K. Itoh, Plasma Phys. Control. Fusion 43, 1055 (2000).

[3] S. Champeaux and P.H. Diamond, Phys. Lett. A. 288, 214 (2001).

[4] B.B. Kadomtsev, Plasma Turbulence (Academic Press, New York, 1965)

[5] R.D. Sydora et al., Plasma Phys. Control. Fusion 38, A281 (1996).

[6] S. Parker et al., Phys. Plasmas 3, 1959 (1996).

[7] X. Garbet et al., Nuclear Fusion 34, 963 (1994).

[8] Z. Lin, S. Ethier, T.S. Hahm et al., Phys. Rev. Lett. 88, 195004 (2002).

[9] Z. Lin, T.S. Hahm, W.W. Lee et al., Phys. Rev. Lett. 83, 3645 (1999).

[10] T.S. Hahm, P.H. Diamond, and Z. Lin, “Turbulence Spreading and Tokamak Transport Scaling” Presented at ITPA Transport/ITB Physics Topical Group Third Meeting, Cadarache, France, October 2002.

[11] Z. Lin, T.S. Hahm, S. Ethier et al., “Size scaling of turbulent transport in tokamak plasmas”, Paper TH/1-1, (IAEA, Lyon, 2002).

[12] For instance, see W. Horton, Rev. Mod. Phys. 71, 735 (1999), and references therein.

[13] P.H. Diamond, V.B. Lebedev, D.E. Newman et al., Phys. Plasmas 2, 3685 (1995).

[14] P.H. Diamond et al., Phys. Rev. Lett. 78, 1472 (1997).

[15] V.B. Lebedev and P.H. Diamond, Phys. Plasmas 4, 1087 (1997).

[16] G.I. Barenblatt, Similarity, Self-similarity, and Intermediate Asymptotics (Consultant Bureau, New York and London, 1979). 
[17] S.-I. Itoh and K. Itoh, J. Phys. Soc. Japan, 69, 408 (2000).

[18] Y. Sarazin, X. Garbet, Ph. Ghendrih et al., Phys. Plasmas 7, 1085 (2000).

[19] Z. Lin, T.S. Hahm, W.W. Lee et al., Science 281, 1835 (1998).

[20] T.S. Hahm, M.A. Beer, Z. Lin et al., Phys. Plasmas 6, 922 (1999).

[21] L. Chen et al.,submitted to Phys. Rev. Lett. (2003).

[22] L. Chen et al., Phys. Plasmas 7, 3129 (2000).

[23] X. Garbet, private communication (2002).

[24] Y. Kishimoto et al., Phys. Plasmas 3, 1289 (1996).

[25] W.W. Lee and R. Santoro, Phys. Plasmas 4, 169 (1997).

[26] P.H. Diamond et al., in Plasma Phys. and Controlled Nuclear Fusion Research (IAEA, Vienna, 1998)IAEA-CN-69/TH3/1.

[27] T.S. Hahm, Plasma Phys. Control. Fusion 44, A87 (2002).

[28] E.J. Kim, P.H. Diamond, M. Malkov et al., Nuclear Fusion 43, 961 (2003).

[29] Z. Lin and T.S. Hahm, "Turbulence spreading and transport scaling in global gyrokinetic particle simulation," submitted to Phys. Plasmas (2003).

[30] H. Biglari, P.H. Diamond, and P.W. Terry, Phys. Fluids B 2, 1 (1990).

[31] T.S. Hahm and K.H. Burrell, Phys. Plasmas 2, 1648 (1995).

[32] A.M. Dimits et al., Phys. Plasmas 7, 969 (2000).

[33] T.S. Hahm, K.H. Burrell, Z. Lin et al., Plasma Phys. Controlled Fusion 42, A205 (2000).

[34] G. Rewoldt and W.M. Tang, Phys. Fluids B 2, 318 (1990).

[35] M. Zarnstorff, Bull. Am. Phys. Soc. 43, 1635 (1998).

[36] T. Fujita et al., Phys. Rev. Lett. 78, 2377 (1997).

[37] B.W. Stallard et al., Phys. Plasmas 6, 1978 (1999). 


\section{External Distribution}

Plasma Research Laboratory, Australian National University, Australia

Professor I.R. Jones, Flinders University, Australia

Professor João Canalle, Instituto de Fisica DEQ/IF - UERJ, Brazil

Mr. Gerson O. Ludwig, Instituto Nacional de Pesquisas, Brazil

Dr. P.H. Sakanaka, Instituto Fisica, Brazil

The Librarian, Culham Laboratory, England

Mrs. S.A. Hutchinson, JET Library, England

Professor M.N. Bussac, Ecole Polytechnique, France

Librarian, Max-Planck-Institut für Plasmaphysik, Germany

Jolan Moldvai, Reports Library, Hungarian Academy of Sciences, Central Research Institute for Physics, Hungary

Dr. P. Kaw, Institute for Plasma Research, India

Ms. P.J. Pathak, Librarian, Institute for Plasma Research, India

Ms. Clelia De Palo, Associazione EURATOM-ENEA, Italy

Dr. G. Grosso, Instituto di Fisica del Plasma, Italy

Librarian, Naka Fusion Research Establishment, JAERI, Japan

Library, Laboratory for Complex Energy Processes, Institute for Advanced Study, Kyoto University, Japan

Research Information Center, National Institute for Fusion Science, Japan

Dr. O. Mitarai, Kyushu Tokai University, Japan

Dr. Jiangang Li, Institute of Plasma Physics, Chinese Academy of Sciences, People's Republic of China

Professor Yuping Huo, School of Physical Science and Technology, People's Republic of China

Library, Academia Sinica, Institute of Plasma Physics, People's Republic of China

Librarian, Institute of Physics, Chinese Academy of Sciences, People's Republic of China

Dr. S. Mirnov, TRINITI, Troitsk, Russian Federation, Russia

Dr. V.S. Strelkov, Kurchatov Institute, Russian Federation, Russia

Professor Peter Lukac, Katedra Fyziky Plazmy MFF UK, Mlynska dolina F-2, Komenskeho Univerzita, SK-842 15 Bratislava, Slovakia

Dr. G.S. Lee, Korea Basic Science Institute, South Korea

Institute for Plasma Research, University of Maryland, USA

Librarian, Fusion Energy Division, Oak Ridge National Laboratory, USA

Librarian, Institute of Fusion Studies, University of Texas, USA

Librarian, Magnetic Fusion Program, Lawrence Livermore National Laboratory, USA

Library, General Atomics, USA

Plasma Physics Group, Fusion Energy Research Program, University of California at San Diego, USA

Plasma Physics Library, Columbia University, USA

Alkesh Punjabi, Center for Fusion Research and Training, Hampton University, USA

Dr. W.M. Stacey, Fusion Research Center, Georgia Institute of Technology, USA

Dr. John Willis, U.S. Department of Energy, Office of Fusion Energy Sciences, USA

Mr. Paul H. Wright, Indianapolis, Indiana, USA 
The Princeton Plasma Physics Laboratory is operated by Princeton University under contract with the U.S. Department of Energy.

\author{
Information Services \\ Princeton Plasma Physics Laboratory \\ P.O. Box 451 \\ Princeton, NJ 08543
}

Phone: 609-243-2750

Fax: 609-243-2751

e-mail: pppl_info@pppl.gov

Internet Address: http://www.pppl.gov 